\title{
Strategy of Fuzzy Mapping using Fixed Point Theorem
}

\author{
J. Madhavi, P. Sree Divya, S. Satya Nagendra Rao
}

\begin{abstract}
To overcome the problems obtained due to non linearity, fixed point theorem. The main intent of this paper is to study the strategy of the fuzzy mapping using fixed point theorem. This fixed point theorem is mainly based on the topological vector space under the fixed selection. This fixed point theorem has convex structure which is in generalized way. This is used in various application fields. In this unity parity function is used to select the spaces available in fuzzy mapping process. The fixed point theorem using fuzzy mapping will improve and extend the classical results.
\end{abstract}

KEYWORDS: Fixed Point Theorem, Transfer Open Valued, Unity Partition, Metric spaces.

\section{INTRODUCTION}

$\mathrm{B}$ asically, large amount of data is transmitted and received in processing applications. The convex values are closed while mapping the semi fixed values. This is first introduced by the Michael in 1965 . There will be fixed selection in mapping process and it will transform from Para compact space to a Banach space. Convex values and open fibers are compacted based on the topological vector space. This is compacted based on the fixed selection. Fixed point theorem will overcome the problems of nonlinearity obtained in the system [1]. Fixed point theorem is used in various applications and directions. There are sub admissible values available in the fixed point theorem for mapping procedure. Hyper convex metric spaces are used to determine the mapping procedure [2].Topological vector space is introduced in the fixed point theorem and there will be an existence of fixed space. By setting the fixed spaces, the fixed theorem is established. There will be combination of both topological vector space and convex vector space. But all these are used in special cases only.

The main intent of this paper is to improve the fixed point theorem using fuzzy mapping [3]. In fuzzy mapping procedure fixed spaces are used. The concept fuzzy mapping was first introduced by the Heilpem. There are fixed points in the fuzzy mapping process. In fuzzy mapping process, the solutions are obtained by using r-degree fixed points and non-less $r$ degree points. To get the exact solutions, the $r$ values are set to 1 . Various types of mappings are introduced, they are non expansive mapping, convex mappings. Among

Revised Manuscript Received on December 12, 2019

Mrs. J. Madhavi, Malla Reddy Engineering College for Women, UGC Autonomous Institution, Maisammaguda, Hyderabad, India

Dr. P. Sree Divya, Malla Reddy Engineering College for Women, UGC

Mr. S. Satya Nagendra Rao Malla Reddy Engineering College for Women, UGC Autonomous Institution, Maisammaguda, Hyderabad, India Autonomous Institution, Maisammaguda, Hyderabad, India

them the fixed point theorem uses the $\mathrm{r}$ degree mapping and non-less $r$ degree mapping [4-5].

All the values of real numbers in metric spaces are replaced in the cone form. The convergence is obtained in the cone metric spaces which will complete the notation in effective way. There is some contract type of mappings available in the cone metric spaces in fixed point theorem. In cone metric spaces there are some self spaces are available based on the contraction pair. Hence in the previous sections, the complete overview of the fixed point theorem using fuzzy mapping is discussed in detail manner

\section{NOTATIONS AND DEFNITIONS}

There are some notations and definitions related to the fixed point theorem. In this section the notations and definitions are described. Consider a non empty set and this is represented as $\mathrm{X}$. The finite sub set for non empty set is given as $X$ 2. $|A|$ is represented for $A \in\langle X\rangle$ which is cardinally equal to $\mathrm{A}$. standard $\mathrm{n}$-simplex is represented by using $\Delta \mathrm{n}$. The vertices used in this $\Delta \mathrm{n}$ are given as $(\mathrm{x}, \mathrm{y})$. The unit vector for this $\Delta \mathrm{n}$ is equal to $\mathrm{n}+1$ for $(\mathrm{i}+1)$ th factor.

The topological space is represented as the subset of T. int $\mathrm{T}$ is given by the interior set for $\mathrm{m}$ of topological space. The notations described in the below as shown:

\section{Definition 1:}

(1) The fuzzy set on $Y$ is introduced when the topological space $\mathrm{Y}$ is maintained to a limit $[0,1] . \Psi(\mathrm{Y})$ will represent the family of fuzzy sets.

(2) Fuzzy mapping is obtained when the topological spaces $X$ and $\mathrm{Y}$ are mapped on the $\mathrm{F}$. this is mapped from $\mathrm{X}$ to $\Psi(\mathrm{Y})$. (3) Degree of membership is nothing but there is a relationship for fuzzy mapping using fuzzy set and number $\mathrm{F}(\mathrm{y})$. The condition used for this mapping is given as $\mathrm{F}: \mathrm{X} \rightarrow$ $\Psi(\mathrm{Y})$.

(4) Strong $\alpha$-cut is introduced which is nothing but a fuzzy set which is maintained at condition $A \in \Psi(Y)$ using the set $(\mathrm{X}, \mathrm{Y})$. The limits for this condition are given as $\alpha \in[0,1)$.

Definition 2: In this fuzzy space are introduced using the vertices $(\mathrm{E}, \mathrm{F})$. The condition maintained for this fuzzy space is given as $\forall N=x \in\langle X\rangle n\{E, F\}$. Here the elements which are obtained will be same and there should be a continuous mapping in the procedure. Let $\mathrm{X}$ is a subset for two vertices $\mathrm{E}$ and $\mathrm{F}$. 


\section{Strategy of Fuzzy Mapping Using Fixed Point Theorem}

Definition 3:

Here $\mathrm{X}$ and $\mathrm{Y}$ are the two vertices of fuzzy metrics. Here $\mathrm{X}$ is nothing but a non empty set and $\mathrm{Y}$ is nothing but a topological space. The open value $\mathrm{X}$ is transferred based on the mapping procedure. The open set value is nothing but a transfer opened value. But in general view they are not inverse.

\section{FUZZY MAPPING USING FIXED POINT THEOREM}

The non empty interior is represented by using a cone as $p$ and the cone metric space is represented as $(\mathrm{x}, \mathrm{d})$ variables.

$\mathrm{X}$ is mapped using self maps depend on the two variable (f, $\mathrm{g})$. The constant $(0,1)$ is maintained and condition for generalized fuzzy mapping is given as

$p(x, y) \epsilon\left\{d\left(x_{v} y\right), d\left(x_{v} f x\right), d(y, g y), \frac{d\left(x_{v}, g y\right)+d\left(y_{v} f x\right)}{2}\right\}$

Such that

$$
d(f x, g y) \leq k p(x, y)
$$

Here $\mathrm{x}$ is the subset of fuzzy space and contraction pair of fuzzy mapping is given as ( $\mathrm{f}, \mathrm{g})$. The both $\mathrm{x}$ and $\mathrm{y}$ are the variables of fuzzy mapping which are obtained from the topological spaces.

Here a cone metric space is considered using the vector spaces $(X, d)$. This is done based on the contraction pair of $X$ subset. A common fixed point is introduced which is very unique in nature. The contraction pair will provide the fuzzy mapping which is equal to the self maps concept and it is given from variables $(x, d)$. Basically, the quasi generalized contraction pair is nothing but a generalized contraction pair.

The cone metric space is represented as $(\mathrm{x}, \mathrm{d})$ and the pair of self maps is represented as ( $\mathrm{f}, \mathrm{g}$ ). The quasi generalized contraction pair ( $\mathrm{f}, \mathrm{g}$ ) is relying on the value of $\mathrm{X}$ which is a subset. $(0,1)$ is the limit maintained for the condition to hold the vector spaces.

\section{(i) $d(f x, g f x) \leq \delta d(x, f x)$ and}

\section{(ii) $d(g x, f g x) \leq \delta d(x, g x)$ for all $x \in X$.}

As earlier discussed that the quasi generalized contraction pair is also known as the generalized contraction pair. Here the points which are obtained from the fixed level have same set $\mathrm{f}$ and $\mathrm{g}$. This concept is mainly introduced by the Lemma and the below shows the description for the generalized contraction pair.

A single ton is obtained because of using the generalized contraction pair ( $f, g$ ). The generalized contraction pair of quasi is described as shown below

$$
\begin{aligned}
& f: X \rightarrow X \text { by } f(x)=x^{2} \text { if } x \in X \text { and } \\
& x \neq 0, f(0)=\frac{1}{4} \text { and put } g=f
\end{aligned}
$$

Here the pair ( $\mathrm{f}, \mathrm{g}$ ) is known as the quasi generalized contraction pair but this is not equal to the generalized contraction pair. The main reason behind this is, there will be no common fixed points in the generalized contraction pair but in quasi generalized contraction pair, there will be contraction pair.

The contraction pair $\mathrm{f}$ and $\mathrm{g}$ is obtained based on the fuzzy mapping of non empty set $\mathrm{X}$.

A non empty set $\mathrm{X}$ for fuzzy mapping is obtained by using the contraction pair $\mathrm{f}$ and $\mathrm{g}$. Hence the sequence for these steps are described below:

$$
\text { In general } x_{2 n+1}=f\left(x_{2 n}\right) \text { and }
$$

$$
x_{2 n+2}=g\left(x_{2 n+1}\right), \quad \text { for } n=0,1,2 \ldots \ldots
$$

For a subset $\mathrm{X}$, the cone metric space should be a quantized generalized contraction pair. The following shows the description for mapping procedure:

(i) Cauchy sequence should be used in the subset $\mathrm{A}$ and after the convergent is performed on the subset $\mathrm{X}$.

(ii) The fixed point set will remain non empty when the functions of $\mathrm{f}$ and $\mathrm{g}$ will have continues flow. The below shows the representation of sequence for mapping procedure.

$$
\begin{aligned}
& \text { (a) } d\left(x_{2 n}, x_{2 n+1}\right) \leq \delta^{2 n} d\left(x_{0}, x_{1}\right) \text { and } \\
& \text { (b) } d\left(x_{2 n+1}, x_{2 n+2}\right) \leq \delta^{2 n+1} d\left(x_{0}, x_{1}\right) \text { for }
\end{aligned}
$$

\section{the sequence $\left\{x_{n}\right\}$ denoted}

\section{by (A)in $X$ and $\delta \in(0,1)$}

satisfying (i) and (ii) of Definition.To see this,

$$
\begin{aligned}
& \text { we have that } d\left(x_{2 n}, x_{2 n+1}\right) \\
& =d\left(x_{2 n-1}, f x_{2 n}\right)
\end{aligned}
$$

\section{(by the definition of the sequence $\left.\left\{x_{n}\right\}\right)$}

$$
\begin{aligned}
& =d\left(g x_{2 n-1}, f g x_{2 n-1}\right) \leq \delta d\left(x_{2 n-1}, g x_{2 n-1}\right) \\
& =d\left(f x_{2 n-2}, g x_{2 n-2}\right) \leq \delta^{2} d\left(x_{2 n-2}, x_{2 n-1}\right)
\end{aligned}
$$

After the process of mapping the following procedure is performed and solutions are obtained they are:

(a) The solution is obtained after the finite number of stets.

(b) The mapping is established very easily.

The below equations shows the representation of Cauchy sequence to get positive integer. The sub set value $\mathrm{X}$ is most widely perform its operation effectively and to perform mapping process easily.

$$
d\left(x_{n}, x_{n+1}\right) \leq \delta^{n} d\left(x_{0}, x_{1}\right) \text { for any } n \geq 1
$$

Let $n, m$ bepositive integers such that $n<m$. 
Then $d\left(x_{n}, x_{m}\right) \leq d\left(x_{n}, x_{n+1}\right)$

$+d\left(x_{n+1}, x_{n+2}\right)+\ldots \ldots \ldots$

$\ldots+d\left(x_{m-1}, x_{m}\right) \leq \delta^{n} d\left(x_{0}, x_{1}\right)$

$+\cdots+\delta^{m-1} d\left(x_{0}, x_{1}\right)$

(by B)

$=\left(\delta^{n}+\delta^{n+1}+\cdots+\delta^{m-1}\right) d\left(x_{0}, x_{1}\right)$

$\leq\left\{a^{a^{n}}\right\} d\left(x_{0}, x_{1}\right) \rightarrow 0$ as $n \rightarrow \infty \ldots$

since $0<\delta<1$.

Here the $\mathrm{Xn}$ is the nothing but a Cauchy sequence. The space $\mathrm{X}$ and $\mathrm{Xn}$ are the convergent at some point on $\mathrm{X}$. if the value of $\mathrm{f}$ is continuous then $\mathrm{f}(\mathrm{x})$ is defined

$f(x)=f\left(\lim x_{2 n}\right)=$

$\operatorname{limf}\left(x_{2 n}\right)=\lim x_{2 n+1}=x$

The fixed point of $\mathrm{f}$ is non empty because the $\mathrm{x}$ is equal to the fixed point of set of $f$. This concept is observed for the lemma that is non empty set will be there in fixed point set. $\mathrm{f}$ and $\mathrm{g}$ are contraction pair variables. Hence, if the value of $g$ is set to be continuous then the value of $\mathrm{f}$ is fixed at certain point. Therefore the fixed sets of $f$ and $g$ are set to no empty.

If $\mathrm{f}=\mathrm{g}=$ identity function on $\mathrm{X}$ then the pair ( $\mathrm{f}, \mathrm{g}$ ) is quasi generalized contraction pair. Further the fixed point sets of $f$ and $g$ are the same and may contain more than one fixed point.

If both $\mathrm{f}$ and $\mathrm{g}$ are not continuous, then the fixed point sets of $\mathrm{f}$ and $\mathrm{g}$ may be empty. This is justified in the following example:

Example : Let $\mathrm{X}=\left[0, \frac{1}{2}\right]$ and $d$ usual metric on $\mathrm{X}$.

Define $f: X \rightarrow X$ by $f(x)=x^{2}$ if $x \in\left(0, \frac{1}{2}\right]$ and $f(0)=\frac{1}{2}$ and put $g=f$.

Then the pair (f, g) is a quasi generalized contraction pair. Also both $f$ and $g$ are not continuous and $f$ and $g$ have no fixed points in $\mathrm{X}$.

\section{CONCLUSION}

The strategy of fixed point theorem using fuzzy mapping is introduced in this paper. This fuzzy mapping procedure consists of fuzzy metric spaces. There are linearity and convexity structures while mapping the fixed point theorem. Hence the fuzzy mapping system gives effectiveness where spaces are introduced.

\section{REFERENCES}

1. J. Markin, "A selection theorem for quasi-lower semi continuous mappings in hyper convex spaces", Journal of Mathematical Analysis and Applications, vol. 321, 2016, pp. 862-866.
2. X.P. Ding, Maximal element theorems in product FC-spaces and generalized games, J. Math. Anal. Appl. 305 (1) (2015) 29-42.

3. W. K. Kim and K. H. Lee, "Generalized fuzzy games and fuzzy equilibrium", Fuzzy Sets and Systems, vol. 122, 2011, pp. 291-301.

4. X. P. Ding and J. Y. Park, "Continuous selection theorem, coincidence theorem, and generalized equilibrium in L-convex spaces", Computers and Mathematics with Applications, vol. 44, 2012, pp. 95-103

5. G. Q. Tian, "Generalization of the FKKM Theorem and the KyFan minimax inequality, with applications to maximal elements, price equilibrium, and complementarily", Journal of Mathematical Analysis and Applications, vol. 170(2), 2008, pp. 457-471.

6. S. Park, "Continuous selection theorems in generalized convex spaces", Numerical Functional Analysis and Optimization, vol. 20, 1999, pp 567-583.

7. H. Ben-Ei-Mechaiekh, S. Chebbi, M. Flornzano, and J. V. Linares, "Abstract convexity and fixed points", Journal of Mathematical Analysis and Applications, vol. 222, 1998, pp. 138-150.

8. X. Wu and S. Shen, "A further generalization of YannelisPrabhakar's continuous selection theorem and its applications", Journal of Mathematical Economics, vol. 197, 1996, pp. 61-74.

9. E. Tarafdar, "Fixed-point theorems in H-spaces and equilibrium point of abstract economies", Journal of the Australian Mathematical Society, Series A, vol. 53, 1992, pp. 252-260.

10. N. C. Yannelis and N. D. Prabhakar, "Existence of maximal elements and equilibria in linear topological spaces", Journal of Mathematical Economics, vol. 12, 1983, pp. 233-245.

11. E. Michael, "Continuous selection I", Annals of Mathematics, vol. 63 , 1981, pp. 361-382

12. F. E. Browder, "A new generalization of the Schauder fixed-point theorem", Mathematics Annalen, vol. 174, 1967, pp. 285-290 